University of Nebraska - Lincoln

DigitalCommons@University of Nebraska - Lincoln

Faculty Publications from the Department of Electrical \& Computer Engineering, Department Electrical and Computer Engineering

2011

\title{
A Hybrid Battery Model Capable of Capturing Dynamic Circuit Characteristics and Nonlinear Capacity Effects
}

Taesic Kim

University of Nebraska-Lincoln, taesickim@huskers.unl.edu

Wei Qiao

University of Nebraska-Lincoln, wqiao@engr.unl.edu

Follow this and additional works at: https://digitalcommons.unl.edu/electricalengineeringfacpub

Part of the Electrical and Computer Engineering Commons

Kim, Taesic and Qiao, Wei, "A Hybrid Battery Model Capable of Capturing Dynamic Circuit Characteristics and Nonlinear Capacity Effects" (2011). Faculty Publications from the Department of Electrical and Computer Engineering. 211.

https://digitalcommons.unl.edu/electricalengineeringfacpub/211

This Article is brought to you for free and open access by the Electrical \& Computer Engineering, Department of at DigitalCommons@University of Nebraska - Lincoln. It has been accepted for inclusion in Faculty Publications from the Department of Electrical and Computer Engineering by an authorized administrator of DigitalCommons@University of Nebraska - Lincoln. 


\title{
A Hybrid Battery Model Capable of Capturing Dynamic Circuit Characteristics and Nonlinear Capacity Effects
}

\author{
Taesic Kim, Student Member, IEEE, and Wei Qiao, Member, IEEE
}

\begin{abstract}
A high-fidelity battery model capable of accurately predicting battery performance is required for proper design and operation of battery-powered systems. However, the existing battery models have at least one of the following drawbacks: 1) requiring intensive computation due to high complexity; 2) not applicable for electrical circuit design and simulation; and 3) not capable of accurately capturing the state of charge (SOC) and predicting runtime of the battery due to neglecting the nonlinear capacity effects. This paper proposes a novel hybrid battery model, which takes the advantages of an electrical circuit battery model to accurately predicting the dynamic circuit characteristics of the battery and an analytical battery model to capturing the nonlinear capacity effects for the accurate SOC tracking and runtime prediction of the battery. The proposed battery model is validated by the simulation and experimental studies for the single-cell and multicell polymer lithium-ion batteries, as well as for a lead-acid battery. The proposed model is applicable to other types and sizes of electrochemical battery cells. The proposed battery model is computational effective for simulation, design, and real-time management of battery-powered systems.
\end{abstract}

Index Terms-Battery model, electrical circuit characteristics, nonlinear capacity effects, rate capacity effect, recovery effect, state of charge (SOC).

\section{INTRODUCTION}

B ATTERIES have been more and more pervasively used as the energy storage and power source for various electrical systems and devices, such as communication systems, electronic devices, renewable power systems, electric vehicles, etc. The proper design and operation of these battery-powered systems and devices requires an appropriate battery model. For example, modern battery power management systems rely on a high-fidelity battery model to track the state of charge (SOC) and predict runtime of each battery cell and the whole battery system to optimize its performance. This requires that the battery model can accurately capture various nonlinear capacity effects of the battery. Moreover, the proper design of a battery-

Manuscript received February 20, 2011; revised July 9, 2011; accepted August 22, 2011. Date of publication October 10, 2011; date of current version November 23, 2011. This work was supported in part by the U.S. National Science Foundation under CAREER Award ECCS-0954938. Paper no. TEC00090-2011.

The authors are with the Department of Electrical Engineering, University of Nebraska-Lincoln, Lincoln, NE 68588-0511 USA (e-mail: taesickim@ huskers.unl.edu; wqiao@engr.unl.edu).

Color versions of one or more of the figures in this paper are available online at http://ieeexplore.ieee.org.

Digital Object Identifier 10.1109/TEC.2011.2167014 powered electrical system or device requires the battery model to be capable of accurately capturing the dynamic electrical circuit characteristics of the battery to facilitate the system-level circuit design and simulation.

A variety of battery models have been developed to capture the battery performance for various purposes, such as battery design, performance estimation/prediction for real-time power management, and circuit simulation. In general, the existing battery models can be classified into four categories [1]: electrochemical models, analytical models, stochastic model, and electrical circuit models. The electrochemical models use complex nonlinear differential equations to exactly describe chemical processes that take place in cells of batteries. For example, Doyle's electrochemical model consists of six coupled, nonlinear differential equations [2]. The electrochemical models are the most accurate models. However, establishing these models requires detailed knowledge of the battery chemical processes, which makes them difficult to configure [1]. Moreover, due to high complexity and intensive computation requirement, it is difficult to use these models for real-time battery power management and circuit simulation.

The analytical models are the simplified electrochemical models that can capture nonlinear capacity effects and predict runtime of the batteries with reduced order of equations. These models perform well for the SOC tracking and runtime prediction under specific discharge profiles. The simplest analytical model is called Peukert's law [3]. It captures the nonlinear relationship between the runtime of the battery and the rate of discharge, but the recovery effect is not taken into account. Another analytical model is the kinetic battery model (KiBaM) proposed in [4] and [5]. The KiBaM is an intuitive battery model, which was originally developed to model chemical processes of large lead-acid batteries by a kinetic process [4]. The third analytical model is the diffusion model, which was developed to model lithium-ion batteries based on the diffusion of the ions in the electrolyte [6]. The model describes the evolution of the concentration of the electroactive species in the electrolyte to predict the battery runtime under a given load profile. The KiBaM and the diffusion model take into account both the rate capacity effect and the recovery effect. However, they cannot describe the current-voltage $(I-V)$ characteristics that are important for electrical circuit simulation and multicell battery design. The KiBaM is actually a first-order approximation of the diffusion model [1].

The stochastic model [7]-[12] focuses on modeling recovery effect and describes the battery behavior as a Markov process 
with probabilities in terms of parameters that are related to the physical characteristics of an electrochemical cell. A stochastic $\mathrm{KiBaM}$ was developed to model a nickel-metal hydride (NiMH) battery [9], where the probability to recover during idle periods is made dependent on the length of the idle periods because the runtime of NiMH batteries strongly depends on the frequency of the load current. The stochastic battery model in [12] gives a good qualitative description for the behavior of a lithium-ion battery under pulsed discharge. However, the model does not handle arbitrary load profiles with varying discharge currents.

The electrical circuit models use equivalent electrical circuits to capture $I-V$ characteristics of batteries by using the combination of voltage and current sources, capacitors, and resistors. Some of these models can also track the SOC and predict the runtime of the batteries by using sensed currents and/or voltages. The electrical circuit models are good for codesign and cosimulation with other electrical circuits and systems. However, the existing electrical circuit models do not integrate battery nonlinear capacity behaviors, leading to an inaccurate prediction of remaining battery capacity and operating time [13]. The rate capacity effect is taken into account in the electrical circuit model of [14] by using a rate factor in the SOC tracking. Recently, an enhanced circuit-based model was developed [15], [16] by mixing an electrical circuit model [13] with Rakhmatov's diffusion analytical model [6] to include the battery recovery effect. However, due to the high complexity of the diffusion analytical model that enhanced model is highly complex and, therefore, is not feasible for real-time applications, such as real-time performance estimation/prediction for power management of batteries.

This paper proposes a novel hybrid battery model based on an electrical circuit battery model and KiBaM [5]. The KiBaM is capable of capturing nonlinear capacity effects, such as the recovery effect and rate capacity effect, for the accurate SOC tracking and runtime prediction of the battery. Therefore, the proposed hybrid model can accurately capture dynamic electrical circuit characteristics and nonlinear behaviors of batteries for any operating conditions. Furthermore, the proposed model is effective for modeling any electrochemical batteries, such as the lead-acid, nickel-cadmium (NiCd), NiMH, and lithiumion. The proposed hybrid battery model is validated by simulation and experimental studies for the single-cell and multicell lithium-ion batteries, as well as for a lead-acid battery.

\section{RELATED WORK}

\section{A. Electrical Circuit Battery Model}

Fig. 1 illustrates an electrical circuit model [13] for a single battery cell, which consists of two capacitor and resistor $(R C)$ circuits. The $R C$ circuit on the left is used for SOC tracking and runtime prediction for the battery cell, where the selfdischarge resistance $R_{\text {self-discharge }}$ is used to characterize the self-discharge energy loss of the battery cell; the capacitance $C_{\text {capacity }}$ is used to represent the charge stored in the battery cell; the current source $i_{\text {cell }}$ represents the charge/discharge current of the battery cell; the voltage across the capacitance $V_{\mathrm{SOC}}$ varies in the range of $0 \mathrm{~V}$ (i.e., the $\mathrm{SOC}$ is $0 \%$ ) to $1 \mathrm{~V}$ (i.e., the SOC is $100 \%$ ), representing the SOC of the battery cell quan-

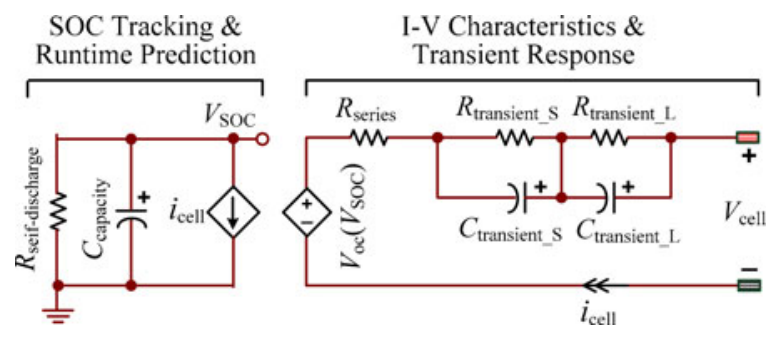

Fig. 1. Electrical circuit battery model.

titatively. The $R C$ circuits on the right simulates the $I-V$ characteristics and transient response of the battery cell, where the voltage-controlled voltage source $V_{\mathrm{OC}}\left(V_{\mathrm{SOC}}\right)$ is used to bridge the SOC (i.e., $V_{\mathrm{SOC}}$ ) to the open-circuit voltage $V_{\mathrm{oc}}$ of the battery cell; the series resistance $R_{\text {series }}$ is used to characterize the charge/discharge energy losses of the battery cell; other resistances and capacitances are used to characterize the short-term (transient_S) and long-term (transient_L) transient responses of the battery cell; and $V_{\text {cell }}$ represents the terminal voltage of the battery cell.

The terminal voltage $V_{\text {cell }}$ of the cell can be determined as follows by the open-circuit voltage $V_{\mathrm{oc}}$ and voltage drop due to the internal impedance $Z_{\mathrm{eq}}$ and current $i_{\text {cell }}$ in Fig. 1:

$$
V_{\text {cell }}=V_{\mathrm{OC}}-i_{\text {cell }} \cdot Z_{\text {eq }} \text {. }
$$

Other than using the left-hand-side $R C$ circuit of Fig. 1, the SOC can also be calculated as [17]

$$
\mathrm{SOC}=\mathrm{SOC}_{\text {initial }}-\int\left(\frac{i_{\text {cell }}}{C_{\text {usable }}}\right) d t
$$

where $\mathrm{SOC}_{\text {initial }}$ is the initial SOC of the cell and $C_{\text {usable }}$ is the usable capacity of the cell. The open-circuit voltage and $R C$ parameters of the model depend on the SOC [13].

The electrical circuit model is relatively accurate to capture the dynamic circuit characteristics of a battery cell, such as the open-circuit voltage, terminal voltage, transient response, and self-discharge. However, this model is unable to capture the nonlinear capacity behaviors, such as the rate capacity effect and recovery effect, of the battery due to the use of a constant capacitance $C_{\text {capacity }}$ to represent the remaining usable capacity of the battery. This reduces the model accuracy when predicting the battery performance at various load current conditions.

\section{B. Kinetic Battery Model}

The rate capacity effect is that less charge can be drawn from a battery when the discharge current is increased. However, the unavailable charge due to a large discharge current still leaves behind in the battery if the thermal dissipation is assumed to be zero. The unavailable charge will become available after a period with no or a low current. This is the recovery effect [9].

One of the models well suited for capturing the nonlinear capacity behaviors of batteries is the $\mathrm{KiBaM}$, which is an intuitive and simple analytical model. The KiBaM describes the chemical processes of a battery by a kinetic process. It assumes that a battery has two charge wells, where the charge is distributed with a capacity ratio $c(0<c<1)$, as shown in Fig. 2. The 


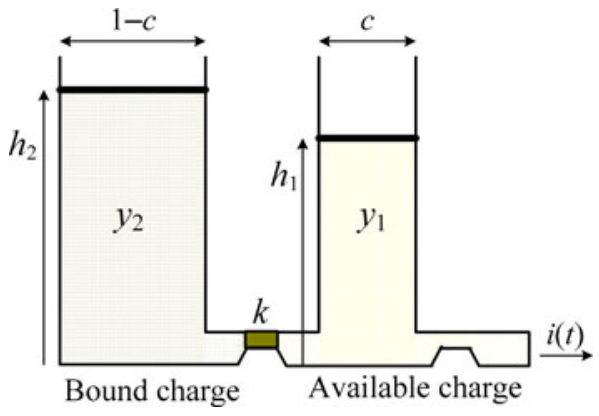

Fig. 2. Kinetic battery model (KiBaM).

available charge well delivers charge directly to the load, while the bound charge well supplies charge only to the available charge well through a valve $k$. The rate of charge flows from the bound charge well to the available charge well depends on $k$ and the difference in heights of the two wells $h_{1}$ and $h_{2}$, where $h_{1}$ represents the SOC of the battery. The battery is fully discharged when $h_{1}$ becomes zero. The change of the charges in the two wells is expressed as [1]

$$
\left\{\begin{array}{l}
\frac{d y_{1}(t)}{d t}=-i(t)+k\left[h_{2}(t)-h_{1}(t)\right] \\
\frac{d y_{2}(t)}{d t}=-k\left[h_{2}(t)-h_{1}(t)\right]
\end{array}\right.
$$

where $y_{1}$ and $y_{2}$ are the total charges in the available charge well and the bound charge well, respectively; $h_{1}=y_{1} / c$; and $h_{2}$ $=y_{2} /(1-c)$. When the battery is discharged with a current of $i(t)$, the available charge reduces faster than the bound charge and the difference in heights of the two wells grows. When the current is removed or reduced, the charge flows from the bound charge well to the available charge well until $h_{1}$ and $h_{2}$ are equal. Therefore, during an idle period or a small-current load, more charge becomes available effectively in the available charge well than when a large-current load is applied continuously. This explains both the recovery effect and rate capacity effect of the battery. Assume the initial conditions of $y_{1,0}=y_{1}\left(t_{0}\right)=$ $c \cdot C, y_{2,0}=y_{2}\left(t_{0}\right)=(1-c) \cdot C$, and $y_{0}=y_{1,0}+y_{2,0}$, where $C$ is the total battery capacity, the differential equations (3) can be solved for a constant discharge current of $I$ for a period of $t_{0} \leq t$ $\leq t_{1}$ by using the Laplace transform; the solutions are given as

$$
\left\{\begin{array}{c}
y_{1}(t)=y_{1,0} e^{-k^{\prime}\left(t-t_{0}\right)}+\frac{\left(y_{0} k^{\prime} c-I\right)\left[1-e^{-k^{\prime}\left(t-t_{0}\right)}\right]}{k^{\prime}} \\
\quad-\frac{I c\left[k^{\prime}\left(t-t_{0}\right)-1+e^{-k^{\prime}\left(t-t_{0}\right)}\right]}{k^{\prime}} \\
y_{2}(t)=y_{2,0} e^{-k^{\prime}\left(t-t_{0}\right)}+y_{0}(1-c)\left[1-e^{-k^{\prime}\left(t-t_{0}\right)}\right] \\
\quad-\frac{I(1-c)\left[k^{\prime}\left(t-t_{0}\right)-1+e^{-k^{\prime}\left(t-t_{0}\right)}\right]}{k^{\prime}} \\
\delta(t)=h_{2}(t)-h_{1}(t)=\frac{y_{2}(t)}{1-c}-\frac{y_{1}(t)}{c}, \quad t_{0} \leq t \leq t_{1}
\end{array}\right.
$$

where $k^{\prime}(=k /[c(1-c)])$ is a constant related to the diffusion rate and $\delta$ is the height difference between the two wells, which plays an important role in obtaining the nonlinear capacity variation. If the discharge current changes to a different value, $y_{1}, y_{2}$, and $\delta$ will be calculated by (4) with the new current value and initial

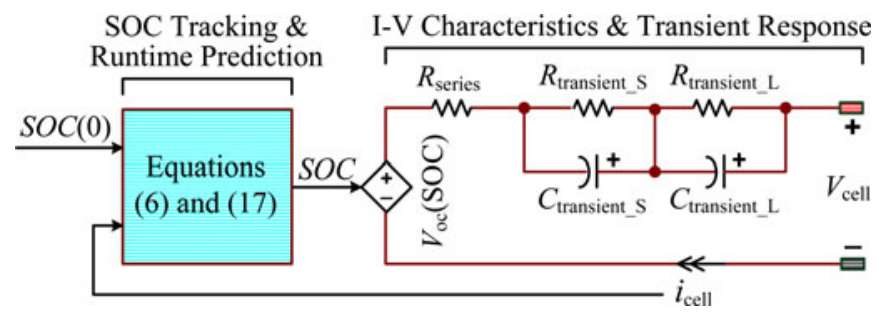

Fig. 3. Proposed hybrid battery model.

conditions of $y_{1,0}$ and $y_{2,0}$, which are the final values of $y_{1}$ and $y_{2}$, respectively, for the previous discharge current. Therefore, (4) can be used to determine $y_{1}, y_{2}$, and $\delta$ for any continuous piecewise constant discharge currents. The discharge completes when $y_{1}$ becomes zero, indicating a zero SOC. Consequently, the unavailable charge $u(t)$ of the battery can be expressed as follows [1]:

$$
u(t)=(1-c) \delta(t) .
$$

The KiBaM model is capable of capturing capacity variation of the battery due to the nonlinear capacity effects. However, it cannot capture the dynamic $I-V$ characteristics of the battery required for codesign and cosimulation with other electrical circuits and systems.

\section{Proposed Hybrid Battery Model}

\section{A. Proposed Hybrid Battery Model}

The proposed hybrid model enhances the electrical circuit model in Fig. 1 by replacing its left-hand-side $R C$ circuit with a module based on the KiBaM to capture the nonlinear capacity variation of the battery, as shown in Fig. 3. Therefore, the proposed model is capable of capturing comprehensive battery performance more accurately than the electrical circuit model in Fig. 1 by coupling the dynamic electrical circuit characteristics with nonlinear capacity effects of the battery. In addition, the proposed battery model needs less computational cost than the enhanced model in [15], thereby is feasible for real-time applications.

Consider a period of $t_{0}<t<t_{r}$ in which the battery cell is first discharged with a constant current (i.e., $i_{\text {cell }}=I>0$ ) and then rests (i.e., $i_{\text {cell }}=0$ ) for the remaining of the period. The proposed battery model is expressed by the following:

$$
\begin{aligned}
& \operatorname{SOC}(t)=\frac{C_{\text {available }}(t)}{C_{\text {max }}} \\
& \quad=\mathrm{SOC}_{\text {initial }}-\frac{1}{C_{\max }}\left[\int i_{\text {cell }}(t) d t+C_{\text {unavaiable }}(t)\right] \\
& V_{\text {oc }}[\operatorname{SOC}(t)]=a_{0} e^{-a_{1} \mathrm{SOC}(t)}+a_{2}+a_{3} \mathrm{SOC}(t) \\
& \quad-a_{4} \mathrm{SOC}^{2}(t)+a_{5} \mathrm{SOC}^{3}(t) \\
& V_{\text {cell }}(t)=V_{\mathrm{OC}}[\mathrm{SOC}(t)]-i_{\text {cell }}(t) \cdot R_{\text {series }}-V_{\text {transient }}(t) \\
& V_{\text {transient }}(t)=V_{\text {transient_S }}(t)+V_{\text {transient_L }}(t)
\end{aligned}
$$




$$
\begin{aligned}
& V_{\text {transient_S }}(t) \\
& \quad= \begin{cases}R_{\text {transient_S }} \cdot i_{\text {cell }}(t)\left[1-e^{-\left(t-t_{0}\right) / \tau_{S}}\right], & t_{0}<t<t_{d} \\
V_{\text {tranisent_S }}\left(t_{d}\right) \cdot e^{-\left(t-t_{d}\right) / \tau_{S}}, & t_{d}<t<t_{r}\end{cases}
\end{aligned}
$$

$$
\begin{aligned}
& V_{\text {transient_L }}(t) \\
& \quad= \begin{cases}R_{\text {transient_L }} \cdot i_{\text {cell }}(t)\left[1-e^{-\left(t-t_{0}\right) / \tau_{L}}\right], & t_{0}<t<t_{d} \\
V_{\text {tranisent_L }}\left(t_{d}\right) \cdot e^{-\left(t-t_{d}\right) / \tau_{L}}, & t_{d}<t<t_{r}\end{cases}
\end{aligned}
$$

where $t_{0}, t_{d}$, and $t_{r}$ are the beginning time, discharge ending time, and (rest) ending time of the period, respectively; $C_{\max }, C_{\text {available, }}$ and $C_{\text {unavailable }}$ are the maximum, available, and unavailable capacities of the battery, respectively; $\tau_{S}$ $=R_{\text {transient_S }} \cdot C_{\text {transient_S }}$; and $\tau_{L}=R_{\text {transient_L }} \cdot C_{\text {transient_L } \mathrm{L}}$. The SOC of the battery reduces when it delivers charge to load, which is expressed by the current integration term in (6). The unavailable capacity $C_{\text {unavailable }}$ represents the nonlinear SOC variation due to the nonlinear capacity effects of the battery. The initial SOC, i.e., $\mathrm{SOC}_{\text {initial, }}$, is the estimated SOC at the end of the last operating period before $t_{0}$. Therefore, to implement the proposed model, only the initial SOC at the beginning of the battery operation (i.e., $t=0$ ) is needed. In practice, $\mathrm{SOC}_{\text {initial }}$ can be corrected by using (7) with the open-circuit voltage measured during some resting time intervals of the battery cell to avoid the accumulation of SOC estimation errors of using (6). Moreover, if the battery operates in the charge mode, the current $i_{\text {cell }}$ becomes negative, leading to the increase of the SOC when using (6).

As in (8), the terminal voltage $V_{\text {cell }}$ is estimated by $V_{\text {oc }}$, the voltage across $R_{\text {series }}\left(\right.$ i.e., $i_{\text {cell }} \cdot R_{\text {series }}$ ), and the transient voltage term $V_{\text {transient }}$, which represents the transient response of the $R C$ network. The $R C$ network parameters are the functions of the SOC:

$$
\left\{\begin{array}{r}
R_{\text {series }}(\mathrm{SOC})=b_{0} e^{-b_{1} \mathrm{SOC}}+b_{2}+b_{3} \mathrm{SOC} \\
-b_{4} \mathrm{SOC}^{2}+b_{5} \mathrm{SOC}^{3} \\
R_{\text {transient_S }}(\mathrm{SOC})=c_{0} \cdot e^{-c_{1} \cdot \mathrm{SOC}}+c_{2} \\
C_{\text {transient_S }}(\mathrm{SOC})=d_{0} \cdot e^{-d_{1} \cdot \mathrm{SOC}}+d_{2} \\
R_{\text {transient_L }}(\mathrm{SOC})=e_{0} \cdot e^{-e_{1} \cdot \mathrm{SOC}}+e_{2} \\
C_{\text {transient_L }}(\mathrm{SOC})=f_{0} \cdot e^{-f_{1} \cdot \mathrm{SOC}}+f_{2} .
\end{array}\right.
$$

These parameters are approximately constant when the SOC is high (e.g., 20-100\% [13]) and change exponentially when the SOC varies below a certain value (e.g., 20-0\% [13]) due to the electrochemical reaction inside the battery. Equations (8)-(11) provide the time-domain response of the $R C$ circuit in Fig. 3.

\section{B. Nonlinear Capacity Variation}

The KiBaM is integrated into the proposed hybrid model to capture the capacity variation of the battery due to nonlinear capacity effects, such as the rate capacity effect and recovery effect. The available capacity $C_{\text {available, which is the remaining }}$ usable capacity in the battery, is determined by

$$
C_{\text {available }}(t)=C_{\text {initial }}-l(t)-C_{\text {unavailable }}(t)
$$

where

$$
l(t)=\int i_{\mathrm{cell}}(t) d t
$$

is the dissipated charge to load at the current of $i_{\text {cell }}$ during the discharge period. The term $C_{\text {unavailable }}(t)$ of (13) represents the unavailable capacity at time $t$, which causes the available capacity to be smaller than the ideal value of $\left[C_{\text {initial }}-l(t)\right]$ due to the rate capacity effect. This effect can be interpreted by the $\mathrm{KiBaM}$ using the available and bound charges.

The unavailable capacity $C_{\text {unavailable }}$ in (13) is determined by the unavailable charge $u(t)$ obtained from the KiBaM model:

$$
C_{\text {unavaiable }}(t)=u(t) \text {. }
$$

A simplified expression for the unavailable charge $u(t)$ can be obtained from (4), given by the following equation:

$$
\begin{aligned}
& u(t)= \\
& \begin{cases}(1-c) \cdot\left[\delta\left(t_{0}\right) e^{-k^{\prime} \cdot\left(t-t_{0}\right)}+\frac{I}{c} \cdot \frac{1-e^{-k^{\prime} \cdot\left(t-t_{0}\right)}}{k^{\prime}}\right], & t_{0}<t<t_{d} \\
(1-c) \delta\left(t_{d}\right) e^{-k^{\prime} \cdot\left(t-t_{d}\right)}, & t_{d}<t<t_{r} .\end{cases}
\end{aligned}
$$

During the discharge time interval $\left(t_{0}<t<t_{d}\right), u(t)$ increases, which represents the rate capacity effect. During the resting time interval $\left(t_{d}<t<t_{r}\right), u(t)$ decreases because the charge flows from the bound charge well to the available charge well, which represents the recovery effect.

Based on (15) and (16), the unavailable capacity $C_{\text {unavailable }}$ can be expressed by (17), shown at the bottom of this page, where $C_{\text {unavailable }}\left(t_{0}\right)$ is zero at $t_{0}=0$. The unavailable capacity determined by (17) enables the proposed model to capture the rate capacity effect during discharge and the recovery effect during rest of the battery. In general, if a battery is discharged with variable and discontinuous currents, then the entire discharge time can be divided into multiple periods, and in each period, the discharge current is constant or zero. Then, (17) can be applied to each period to continuously capture the unavailable capacity of the battery. A special case is the discharge with a continuous constant current or with a constant pulse current.

Fig. 4 shows the simulated nonlinear capacity variation of a 1-Ah, 3.7-V lithium-ion battery cell when it is discharged with a current of $3 \mathrm{C}$ (i.e., $3 \mathrm{~A}$ ) for $500 \mathrm{~s}$ and then rests for $500 \mathrm{~s}$ with zero discharge current, where $k^{\prime}=0.005$ and $c=0.3$ are used for the proposed battery model. In Fig. $4, C_{\text {unavailable increases }}$ over time during the discharge period and reaches the maximum value at time $t_{d}=500 \mathrm{~s}$. After that, $C_{\text {unavailable }}$ reduces during the idle time from $t_{d}=500 \mathrm{~s}$ to $t_{r}=1000 \mathrm{~s}$, indicating that the unavailable capacity gradually becomes available, i.e., recovery

$$
C_{\text {unavailable }}(t)= \begin{cases}C_{\text {unavailable }}\left(t_{0}\right) e^{-k^{\prime} \cdot\left(t-t_{0}\right)}+(1-c) \frac{I}{c} \cdot \frac{1-e^{-k^{\prime} \cdot\left(t-t_{0}\right)}}{k^{\prime}}, & t_{0}<t<t_{d} \\ C_{\text {unavailable }}\left(t_{d}\right) e^{-k^{\prime} \cdot\left(t-t_{d}\right)}, & t_{d}<t<t_{r}\end{cases}
$$




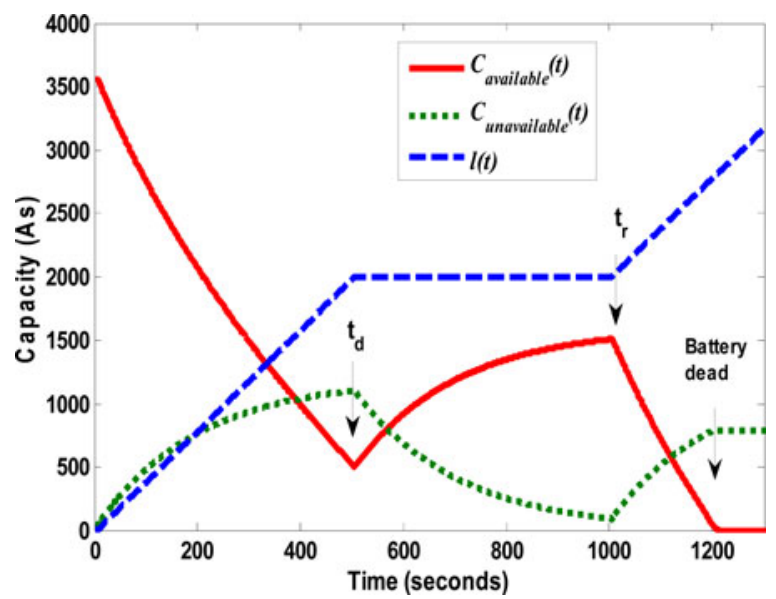

Fig. 4. Nonlinear capacity variation of a 1-Ah lithium-ion battery cell when discharging with $3 \mathrm{C}$ for $500 \mathrm{~s}$ and resting for another $500 \mathrm{~s}$.

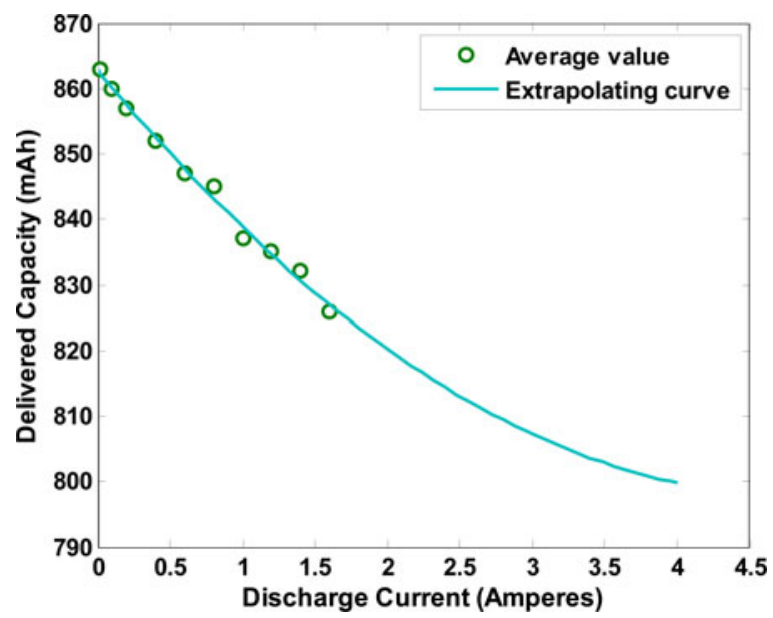

Fig. 5. Maximum available capacity of an $860-\mathrm{mAh}$ polymer lithium-ion battery cell as a function of the discharge current.

of the battery capacity. The battery is fully discharged when $C_{\text {available }}$ becomes zero.

Fig. 5 shows the measured maximum available capacity of an 860-mAh, 3.7-V polymer lithium-ion battery cell (see the appendix), which depends on the discharge current. The extrapolating curve in Fig. 5 illustrates the maximum available capacity under various load conditions. $C_{\text {unavailable }}$ increases as the discharge current increases, which results in the reduction of the available capacity. If the battery cell is discharged to an infinitesimal load, the battery runtime is extremely short. Therefore, there is no time for the charge to move from the bound charge well to the available charge well; the maximum available capacity equals the amount of charge in the available charge well. On the contrary, if the discharge current is small, all of the charges in the bound and available charge wells will become available to be delivered to the load.

\section{Model Extraction}

All of the electrical circuit parameters of the proposed battery model can be extracted from least-squares curve fitting of the

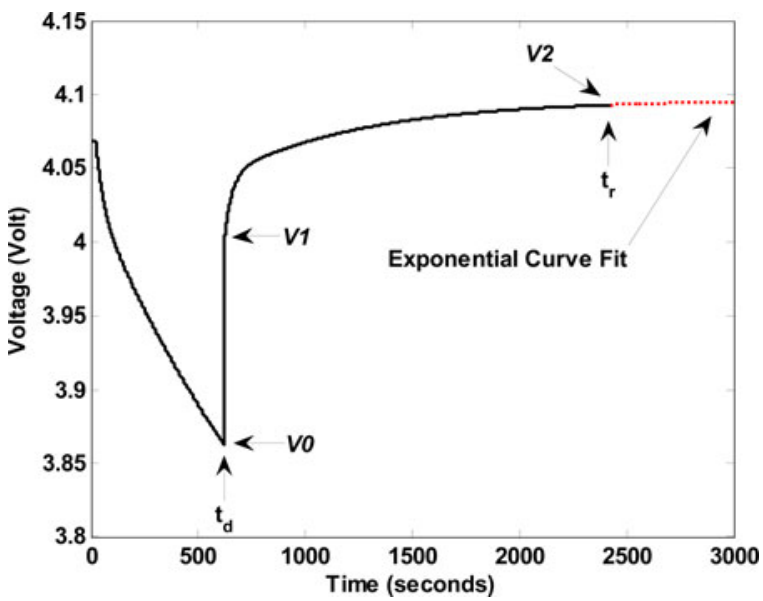

Fig. 6. Typical curve of terminal voltage response under pulsed-current discharge for extraction of the electrical circuit parameters of the proposed battery model.

experiment data obtained at room temperature using pulse discharge currents with an interval of 5\% SOC. In this section, the 860-mAh, 3.7-V polymer lithium-ion battery cell will be used to illustrate how the model is extracted. The experimental procedure to extract $V_{\text {oc }}(\mathrm{SOC})$ and $R_{\text {series }}(\mathrm{SOC})$ is similar to that in [13] and [19]. Fig. 6 shows a typical curve of terminal voltage response used for extraction of the electrical circuit parameters of the proposed model. The battery cell is discharged with a current of $I=0.6 \mathrm{C}$ (i.e., $0.516 \mathrm{~A}$ ) during $0 \leq t \leq t_{d}$ and then rests during $t_{d} \leq t \leq t_{r}$ with zero discharge current. During the time $0 \leq t \leq t_{d}, 5 \% \mathrm{SOC}$ of the battery cell dissipated. Then, the battery cell rests for enough time, e.g., $30 \mathrm{~min}$, to allow it to recover the unavailable capacity. This ensures that the electrical circuit parameters are independent of the rate capacity effect because the SOC tracking part of the model has taken into account that effect. $V_{\mathrm{oc}}(\mathrm{SOC})$ in (7) is extracted by estimating the steady-state open circuit voltage using the exponential curve fitting. Moreover, the instantaneous voltage rising when discharge finished at $t_{d}$ has a relationship with $R_{\text {series }}(\mathrm{SOC})$ in (8), which can be calculated by the following equation:

$$
R_{\text {series }}(\mathrm{SOC})=\frac{V 1-V 0}{I} .
$$

Based on (8)-(11), the following equation is obtained to estimate the $R C$ network parameters:

$$
V_{\text {cell }}(t)=a \cdot\left(1-e^{-b \cdot t}\right)+c \cdot\left(1-e^{-d \cdot t}\right)+e
$$

where $e$ is $V 1 ; V_{\text {cell }}=V_{o c}$ when $t \rightarrow \infty$. The parameter $a, b, c$, and $d$ are determined from the least-squares curve fitting. The $R C$ network parameters can be then derived from

$$
\left\{\begin{array}{l}
R_{\text {Transient_S }}=\frac{a}{I} \\
C_{\text {Transient_S }}=\frac{1}{R_{\text {Transient_S }} \cdot b} \\
R_{\text {Transient_L }}=\frac{c}{I} \\
C_{\text {Transient_L }}=\frac{1}{R_{\text {Transient_L }} \cdot d} .
\end{array}\right.
$$


TABLE I

BATtery Model Parameters For a POLYMER Lithium-ION CELL

\begin{tabular}{|l|l|l|l|l|l|l|l|}
\hline$a_{0}$ & -0.852 & $a_{1}$ & 63.867 & $a_{2}$ & 3.6297 & $a_{3}$ & 0.559 \\
\hline$a_{4}$ & 0.51 & $a_{5}$ & 0.508 & $b_{0}$ & 0.1463 & $b_{1}$ & 30.27 \\
\hline$b_{2}$ & 0.1037 & $b_{3}$ & 0.0584 & $b_{4}$ & 0.1747 & $b_{5}$ & 0.1288 \\
\hline$c_{0}$ & 0.1063 & $c_{1}$ & 62.49 & $c_{2}$ & 0.0437 & $d_{0}$ & -200 \\
\hline$d_{1}$ & -138 & $d_{2}$ & 300 & $e_{0}$ & 0.0712 & $e_{1}$ & 61.4 \\
\hline$e_{2}$ & 0.0288 & $f_{0}$ & -3083 & $f_{1}$ & 180 & $f_{2}$ & 5088 \\
\hline$y_{1,0}$ & $2,863.3$ & $y_{2,0}$ & 232.66 & $c$ & 0.9248 & $k^{\prime}$ & 0.0008 \\
\hline
\end{tabular}

The parameter $c$ and initial conditions $y_{1,0}$ and $y_{2,0}$, are determined from the maximum available charge (i.e., capacity Ah.3600 s) under very large and very small current loads [9]. The delivered capacity under a very small current load is the total initial charge $y_{0}$ of the two charge wells. The maximum available capacity under a very large current load (i.e., the infinitesimal load) is the initial charge $y_{1,0}$ of the available charge well in Fig. 2. Then, the initial charge $y_{2,0}\left(=y_{0}-y_{1,0}\right)$ of the bound charge well and the capacity ratio $c\left(=y_{1,0} / y_{0}\right)$ can be determined. As shown in Fig. 5, the maximum available charge is $3114(=0.865 \mathrm{Ah} \cdot 3600 \mathrm{~s})$, which is $y_{0} ; y_{1,0}$ is $2872.8(=$ $0.798 \mathrm{Ah} \cdot 3600 \mathrm{~s}$ ), where $0.798 \mathrm{Ah}$ is the maximum available charge at the infinitesimal load. Consequently, the value of the parameter $c=0.9248$. The value of $k^{\prime}$ is determined in such a way that the unavailable capacity $C_{\text {unavailable }}$ obtained from (17) agrees with experimental results by discharging the battery cell with continuous constant currents from full SOC until the cutoff voltage is reached. Since $C_{\text {unavailable }}\left(t_{0}\right)$ is zero at $t_{0}=$ $0, t_{d}$ is known, and the value of $c$ has been derived, only $k$ ' is unknown in (17). Therefore, $k$ ' can be extracted. The parameter $k^{\prime}$ of the polymer lithium-ion battery cells is almost constant for any continuous current loads.

\section{Model Validation}

Simulation and experimental studies are carried out to validate the proposed hybrid battery model for a single-cell, as well as a six-cell, polymer lithium-ion battery for various discharge current operations. Comparison with the electrical circuit model in [13] is also provided to show the superiority of the proposed model.

\section{A. Simulation of the Proposed Battery Model}

The proposed hybrid battery model is implemented in MATLAB/Simulink for an 860-mAh, 3.7-V polymer lithium-ion battery cell (see the appendix). The parameters of the single-cell model are obtained by using the model extraction method in Section III-C and are listed in Table I. Based on the singlecell model, a series-connected, six-cell battery pack is built in MATLAB/Simulink. A cell switching circuit proposed in [17] and [18] is employed to control the operation (i.e., charge, discharge, and rest) of each cell independently.

\section{B. Experimental Setup}

The six-cell battery pack simulated in MATLAB/Simulink is constructed in hardware to further validate the proposed model.

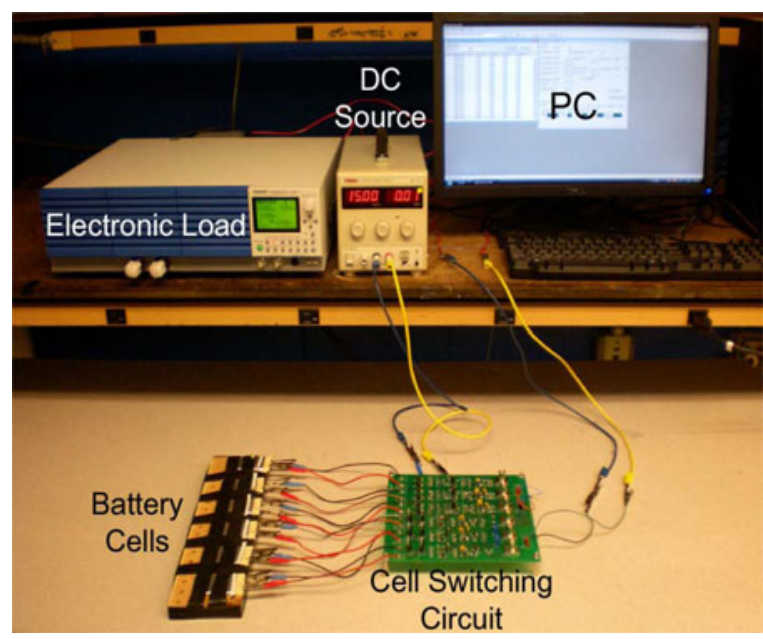

Fig. 7. Experimental setup.

Fig. 7 illustrates the experimental setup. The cells are charged with constant current constant voltage (CCCV) by a dc source and, then, discharged under various discharge current profiles through a programmable dc electronic load, which offers constant resistor (C.R.), constant current (C.C.), and pulse current (P.C.) modes. High-efficiency power MOSFETs are used to construct the cell switching circuit on a printed circuit board (PCB) [17], [18]. The sensing, control, and protection functions are also implemented on the PCB.

\section{Single-Cell Study for a Polymer Lithium-Ion Battery}

Fig. 8 compares the terminal voltage responses obtained from simulations using the electrical circuit model and the proposed hybrid model with experimental results for a single cell for two constant-current discharge scenarios, where the discharge currents are $0.93 \mathrm{C}(0.8 \mathrm{~A})$ and $1.86 \mathrm{C}(1.6 \mathrm{~A})$, respectively. The terminal voltage responses obtained from the proposed model match the experimental results better than those obtained from the electrical circuit model, particularly when the battery cell is close to fully discharged. Therefore, the proposed model can accurately predict the runtimes of the battery cell under various discharge current conditions. However, due to neglecting the rate capacity effect, the runtime prediction errors of the electrical circuit model are obvious and increase significantly as the discharge current increases.

Fig. 9 compares the terminal voltage responses obtained from simulations using the electrical circuit model and the proposed model with experimental results for two pulse-current discharge scenarios, where each current pulse has 600-s ON time and 600-s OFF time. Again, the proposed model captures the dynamic responses and predicts the runtimes of the battery cell accurately under various pulse-current discharge conditions. On the other hand, due to neglecting the rate capacity and recovery effects, the errors of dynamic response tracking and runtime prediction of the electrical circuit model are larger than the proposed model and increase at higher discharge currents.

Furthermore, by capturing the variation of the unavailable capacity due to the recovery effect, the proposed hybrid model 


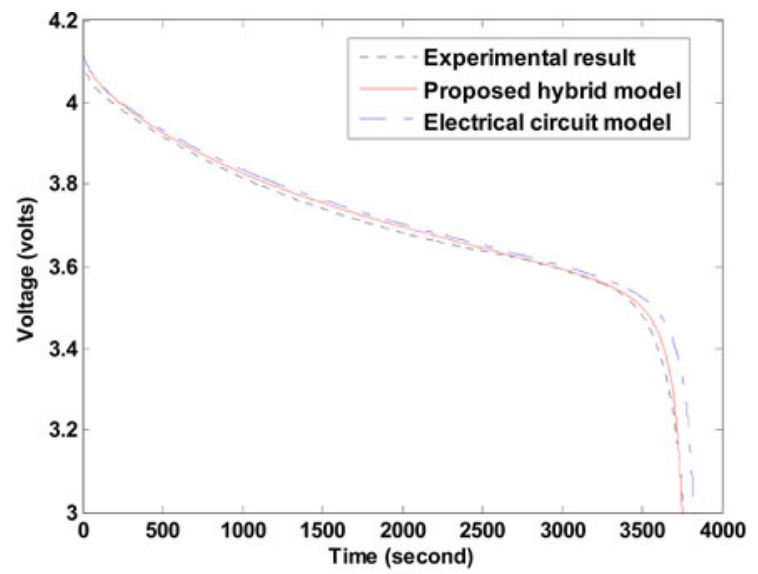

(a)

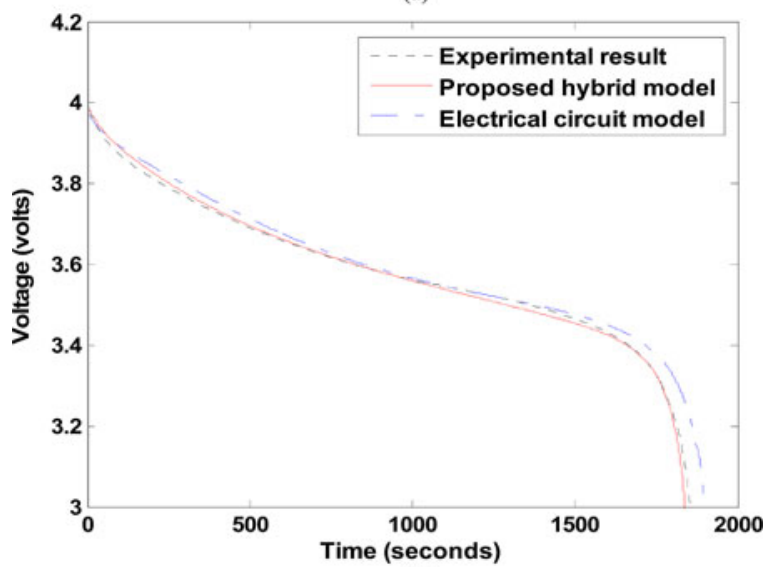

(b)

Fig. 8. Comparison of simulation results of the electrical circuit model and the proposed hybrid model with experimental results for a single-polymer lithiumion cell with constant discharge currents of (a) $0.93 \mathrm{C}(0.8 \mathrm{~A})$ and (b) $1.86 \mathrm{C}$ (1.6 A).

is able to accurately capture the SOC variation of the single cell under the pulse-current discharge, as shown in Fig. 10.

\section{Multicell Study for a Polymer Lithium-Ion Battery}

Experiments are performed at different scenarios to compare with corresponding simulation results to validate the proposed battery model [18] for the six-cell polymer lithium-ion battery, where the dynamics of each cell are represented by the proposed model. For all scenarios, the experimental results agree with the simulation results obtained from the proposed model, as shown in Table II. In Scenario 1, the six cells are discharged using the C.R. mode simultaneously. Since the initial SOCs of the cells are different, the cells are fully discharged sequentially. Once a cell is fully discharged, it will be disconnected from the battery pack by the cell switching circuit but the remaining cells still supply energy to the load. Fig. 11 compares the terminal voltage responses of the six-cell battery obtained from the simulation and experiment for Scenario 1. The results show that not only the steady state but also the dynamic responses of the battery obtained from the simulation agree with those obtained from the experiment.

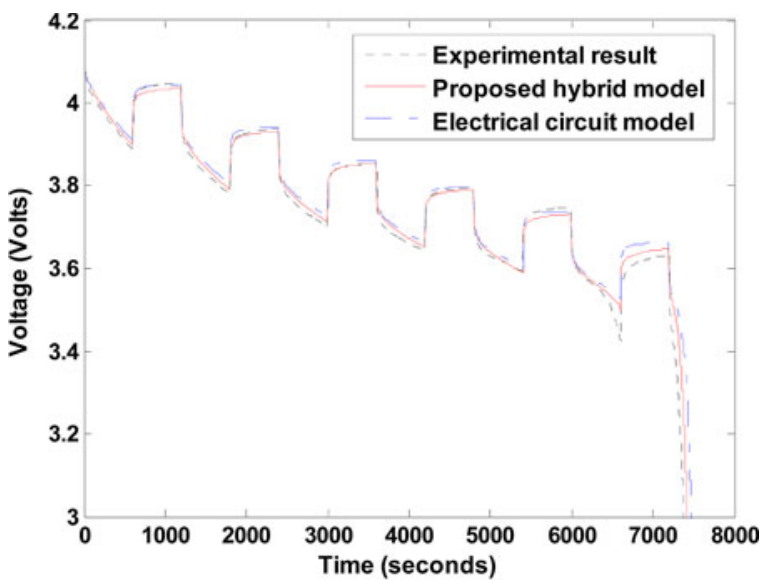

(a)

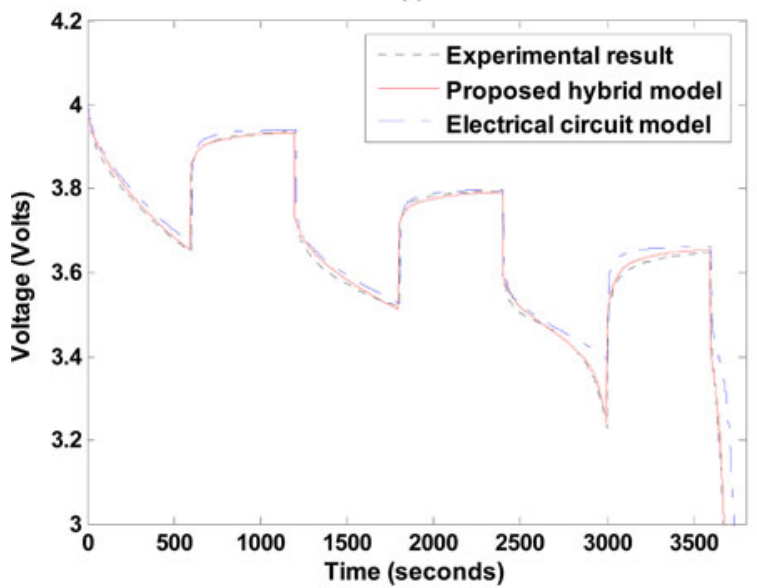

(b)

Fig. 9. Comparison of simulation results of the electrical circuit model and the proposed hybrid model with experimental results for a single-polymer lithiumion cell with pulse discharge currents of (a) $0.93 \mathrm{C}(0.8 \mathrm{~A})$ and (b) $1.86 \mathrm{C}(1.6 \mathrm{~A})$, where each pulse has 600-s ON time and 600-s OFF time.

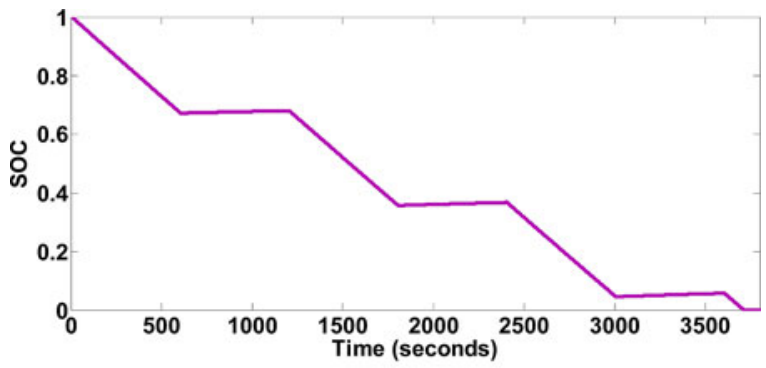

(a)

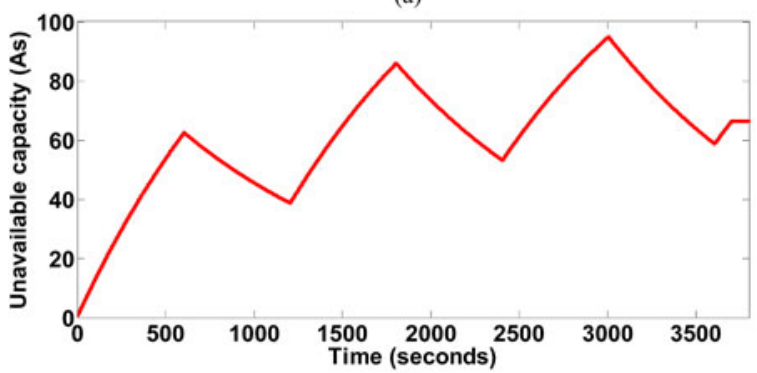

(b)

Fig. 10. Nonlinear capacity variation estimated by the proposed model for a single-polymer lithium-ion cell under a pulse discharge current of $1.86 \mathrm{C}$ (1.6A), where each pulse has 600-s ON time and 600-s OFF time: (a) SOC variation and (b) unavailable capacity. 
TABLE II

COMPARISON OF SimULATION AND EXPERIMENTAL RESULTS FOR THE SiX-CELl BATTERY

\begin{tabular}{|l|l|l|l|l|l|l|l|l|l|}
\hline \multirow{2}{*}{ Scenario } & \multirow{2}{*}{ Discharge method } & \multicolumn{3}{|l|}{ Initial cell conditions expressed by SOC [\%] } & \multicolumn{2}{l|}{ Energy [Wh] } \\
\cline { 3 - 13 } & & Cell 1 & Cell 2 & Cell 3 & Cell 4 & Cell 5 & Cell 6 & Simulation & Experiment \\
\hline 1 & C.R. $=100 \Omega$ & 100 & 80 & 55 & 38 & 13 & 100 & 12.38 & 12.28 \\
\hline 2 & C.C. $=860 \mathrm{~mA}$ & 100 & 100 & 100 & 100 & 100 & 100 & 18.41 & 18.30 \\
\hline 3 & P.C. $=860 \mathrm{~mA}(300 \mathrm{~s}$ on, 300s off) & 100 & 100 & 100 & 100 & 100 & 100 & 18.66 & 18.6 \\
\hline
\end{tabular}

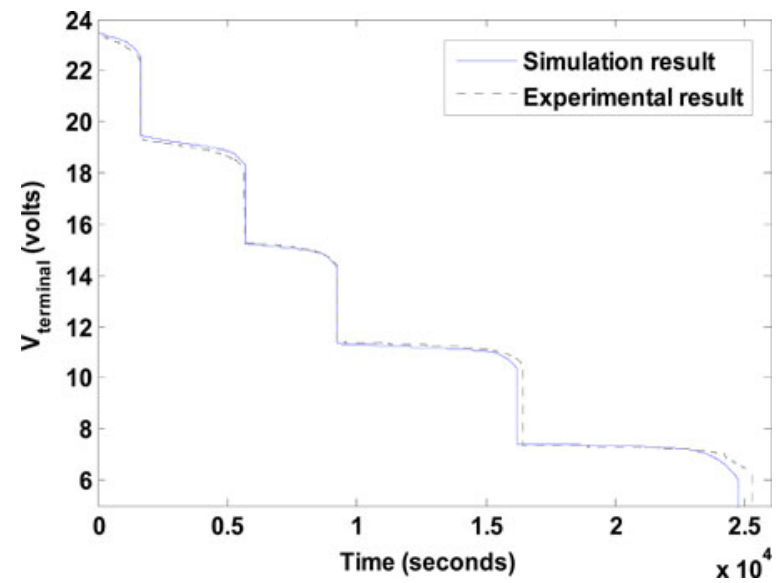

Fig. 11. Comparison of simulation and experimental results in Scenario 1 for the terminal voltage of the six-cell battery.

In Scenario 2, all of the six cells are discharged simultaneously using the C.C. method. In Scenario 3, the six cells are divided into two groups and each group has three cells. The two groups of cells are discharged alternatively, i.e., P.C. discharge, with a time interval of $300 \mathrm{~s}$ until all of the cells are fully discharged. As shown in Table II, compared to using the C.C. discharge (Scenario 2), more energy (300 $\mathrm{mWh}$ ) is supplied by the six-cell battery when using the P.C. discharge (Scenario 3). This P.C. discharge method utilizes the recovery effect to improve the energy-conversion efficiency of the multicell battery. These results show that proposed model can accurately capture the nonlinear capacity variation and dynamic electrical circuit characteristics of each cell as well as the whole battery pack for various discharge modes.

\section{E. Study for a Lead-Acid Battery}

A lead-acid battery was tested to further validate the hybrid battery model. Table III shows the parameters of the battery model extracted by the method described in Section III-C. The capacity ratio $c$ of the lead-acid battery is lower than that of the polymer lithium-ion cell, while $k^{\prime}$ of the lead-acid battery is higher than that of the lithium-ion cell. These parameters indicate that the lead-acid battery has higher nonlinear capacity variations than the lithium-ion battery.

Fig. 12 compares the terminal voltage responses obtained from simulations using the electrical circuit model and the proposed hybrid model with the experimental result for a pulsed discharge scenario, where the battery is discharged with a con-
TABLE III

BATTERY MODEL PARAMETERS FOR A LEAD-ACID BATTERY

\begin{tabular}{|l|l|l|l|l|l|l|l|}
\hline$a_{0}$ & 5.429 & $a_{1}$ & 117.5 & $a_{2}$ & 11.32 & $a_{3}$ & 2.706 \\
\hline$a_{4}$ & 2.04 & $a_{5}$ & 1.026 & $b_{0}$ & 1.578 & $b_{1}$ & 8.527 \\
\hline$b_{2}$ & 0.7808 & $b_{3}$ & -1.887 & $b_{4}$ & -2.404 & $b_{5}$ & -0.649 \\
\hline$c_{0}$ & 2.771 & $c_{1}$ & 9.079 & $c_{2}$ & 0.22 & $d_{0}$ & -2423 \\
\hline$d_{1}$ & 75.14 & $d_{2}$ & 55 & $e_{0}$ & 2.771 & $e_{1}$ & 9.079 \\
\hline$e_{2}$ & 0.218 & $f_{0}$ & -1240 & $f_{1}$ & 9.571 & $f_{2}$ & 3100 \\
\hline$y_{1,0}$ & 2592 & $y_{2,0}$ & 1728 & $c$ & 0.6 & $k^{\prime}$ & 0.0034 \\
\hline
\end{tabular}

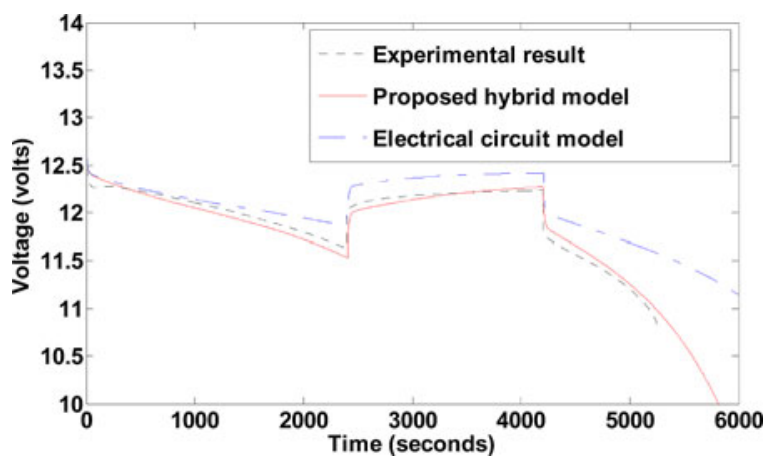

Fig. 12. Comparison of simulation results of the electrical circuit model and the proposed hybrid model with experimental results for a lead-acid battery for a pulsed discharge scenario.

stant current of $0.6 \mathrm{C}(0.74 \mathrm{~A})$ for $40 \mathrm{~min}$, rests for $30 \mathrm{~min}$, and, then, discharged until the cutoff voltage is reached. As shown in Fig. 12, the terminal voltage response obtained from the proposed model matches the experimental result much better than that obtained from the electrical circuit model. The errors of the terminal voltage predicted from the proposed model are less than $1 \%$ with respect to the experimental result. This means that the proposed model continuously tracks the SOC of the battery accurately. Consequently, the runtime of the battery predicted from the proposed model is almost the same as that obtained from the experiment. On the contrary, the error of the runtime predicted from the electrical circuit model is significant. Therefore, the proposed model can accurately capture the dynamic circuit characteristics and nonlinear capacity effects of leadacid batteries as well.

\section{CONCLUSION}

This paper has presented a novel hybrid battery model, which is capable of capturing dynamic electrical circuit characteristics and nonlinear capacity variation of battery cells under various operating conditions. The proposed battery model has been 
implemented in MATLAB/Simulink for a single-cell battery and a six-cell battery using $860-\mathrm{mAh}, 3.7-\mathrm{V}$ polymer lithium-ion cells as well as for a 1.2-Ah, 12-V lead-acid battery. Simulation studies have been performed and compared with experiments to validate the proposed model. Results have shown that the proposed model is able to capture nonlinear capacity effects and dynamic electrical circuit characteristics and predict the runtimes accurately not only for single cell but also for multicell batteries for various discharge modes and load current conditions. Compared to the existing electrical circuit battery models, the proposed hybrid model can offer more accurate SOC tracking and runtime prediction, thereby more accurate dynamic circuit characteristics capturing.

The proposed battery model can be applied to any type and size of electrochemical battery cells, such as lead-acid, NiCd, $\mathrm{NiMH}$, and lithium-ion cells. It provides an accurate model for battery and circuit-system designers to study various battery characteristics and optimally design battery systems for various applications. Moreover, the proposed battery model is computational effective and can be used in battery power management to optimize the energy-conversion efficiency and prolong the operating time of battery systems in real time.

\section{APPENDIX}

The parameters of the polymer lithium-ion battery cells are listed as follows: cell model: pl-383562 2 C; nominal voltage: $3.7 \mathrm{~V}$; nominal capacity: $860 \mathrm{mAh}$; discharge cutoff voltage $V_{\text {cutoff }}: 3 \mathrm{~V}$; charge cutoff voltage $V_{\text {over }}: 4.2 \mathrm{~V}$; and maximum discharge current: $2 \mathrm{C}(1.72 \mathrm{~A})$.

The parameters of the lead-acid battery are listed as follows. Battery model: LEOCH LP12-1.2AH; nominal voltage: $12 \mathrm{~V}$; nominal capacity: $1.2 \mathrm{Ah}$; discharge cutoff voltage $V_{\text {cutoff }}$ : $10.8 \mathrm{~V}$; charge cutoff voltage $V_{\text {over }}: 13.5 \mathrm{~V}$; and maximum discharge current: $15 \mathrm{C}$ (18 A).

\section{REFERENCES}

[1] M. R. Jongerden and B. R. Haverkort, "Which battery model to use?," IET Softw., vol. 3, no. 6, pp. 445-457, Dec. 2009.

[2] M. Doyle, T. F. Fuller, and J. Newman, "Modeling of galvanostatic charge and discharge of the lithium/polymer/insertion cell," J. Electrochem. Soc., vol. 140, no. 6, pp. 1526-1533, Jun. 1993.

[3] D. Linden and T. B. Reddy, Handbook of Batteries, 3rd ed. New York: McGraw-Hill, 2001.

[4] J. Manwell and J. Mcgowan, "Lead acid battery storage model for hybrid energy system," Solar Energy, vol. 50, pp. 399-405, 1993.

[5] J. Manwell and J. Mcgowan, "Extension of the kinetic battery model for wind/hybrid power system," in Proc. 5th Eur. Wind Energy Assoc. Conf., 1994, pp. 1182-1187.

[6] D. Rakhmatov, S. Vrudhula, and A. Wallach, "An analytical high-level battery model for use in energy management of portable electronic systems," in Proc. Int. Conf. Comput. Aided Design, 2001, pp. 488-493.

[7] C. Chiasserini and R. Rao, "Pulsed battery discharge in communication devices," in Proc. 5th Int. Conf. Mobile Comput. Netw., 1999, pp. 88-95.

[8] D. Panigrahi, C. Chiasserini, S. Dey, R. Rao, A. Raghunathan, and K. Lahiri, "Battery life estimation of mobile embedded systems," in Proc. Int. Conf. VLSI Design, 2001, pp. 55-63.

[9] V. Rao, G. Singhal, A. Kumar, and N. Navet, "Battery modeling for embedded systems," in Proc. 18th Int. Conf. VLSI Design, 2005, pp. 105110.

[10] C. Chiasserini and R. Rao, "A model for battery pulsed discharge with recovery effect," in Proc. Wireless Commun. Netw. Conf., 1999, pp. 636639.
[11] C. Chiasserini and R. Rao, "Improving battery performance by using traffic shaping techniques," IEEE J. Sel. Areas Commun., vol. 19, no. 7, pp. 1385-1394, Jul. 2001.

[12] C. Chiasserini and R. Rao, "Energy efficient battery management," IEEE J. Sel. Areas Commun., vol. 19, no. 7, pp. 1235-1245, Jul. 2001.

[13] M. Chen and G. A. Rincon-Mora, "Accurate electrical battery model capable of predicting runtime and I-V performance," IEEE Trans. Energy Convers., vol. 21, no. 2, pp. 504-511, Jun. 2006.

[14] L. Gao, S. Liu, and A. Dougal, "Dynamic lithium-ion battery model for system simulation," IEEE Trans. Compon. Packag. Technol., vol. 25, no. 3, pp. 495-505, Sep. 2002.

[15] J. Zhang, S. Ci, Sharif, and M. Alahmad, "An Enhanced circuit-based model for single-cell battery," in Proc. 25th Appl. Power Electron. Conf. Exhib., Feb. 2010, pp. 672-675.

[16] J. Zhang, S. Ci, Sharif, and M. Alahmad, "Modeling discharge behavior of multicell battery," IEEE Trans. Energy Convers., vol. 25, no. 4, pp. 1133 1141, Dec. 2010.

[17] T. Kim, W. Qiao, and L. Qu, "Series-connected reconfigurable multicell battery: A novel design toward smart batteries," in Proc. IEEE Energy Convers. Congr. Expo., 2010, pp. 4257-4263.

[18] T. Kim, W. Qiao, and L. Qu, "Series-connected self-reconfigurable multicell battery," in Proc. 26th Appl. Power Electron. Conf. Expo., Mar. 2011, pp. 1382-1387.

[19] S. Abu-Sharkh and D. Doerffel, "Rapid test and non-linear model characterization of solid-state lithium-ion batteries," J. Power Sources, vol. 130, pp. 266-274, 2004.

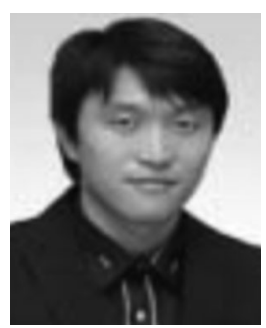

Taesic Kim (S'10) received the B.S. degree in electronics engineering from Changwon National University, Changwon, Korea, in 2008. Currently, he is working toward the Ph.D. degree in electrical engineering at the University of Nebraska-Lincoln, Lincoln, NE.

In 2009, he was with the New and Renewable Energy Research Group of Korea Electrotechnology Research Institute, Changwon, Korea. His research interests include renewable energy systems, power electronics, battery modeling and power management, and energy storage system design and optimization.

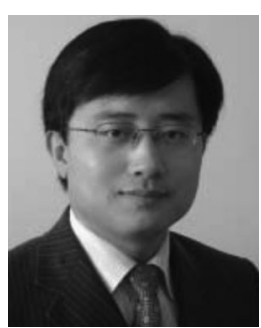

Wei Qiao (S'05-M'08) received the B.Eng. and M.Eng. degrees in electrical engineering from Zhejiang University, Hangzhou, China, in 1997 and 2002, respectively, the M.S. degree in high-performance computation for engineered systems from SingaporeMIT Alliance, Singapore, in 2003, and the Ph.D. degree in electrical engineering from Georgia Institute of Technology, Atlanta, in 2008.

From 1997 to 1999, he was an Electrical Engineer with China Petroleum and Chemical Corporation (Sinopec). Since 2008, he has been an Assistant Professor of Electrical Engineering with the University of Nebraska-Lincoln (UNL), Lincoln, NE. He is the author or coauthor of three book chapters and more than 60 papers in refereed journals and international conference proceedings. His research interests include renewable energy systems, smart grid, power system control and optimization, condition monitoring and fault diagnosis, energy storage, power electronics, electric machines and drives, and computational intelligence for electric power and energy systems.

Dr. Qiao is an Associate Editor of the IEEE TRANSACTIONS ON INDUSTRY ApPLICATIOS, the Chair of the Technical Thrust of Sustainable Energy Sources of the IEEE Power Electronics Society, and the Chair of the Task Force on Intelligent Control for Wind Plants of the IEEE Power and Energy Society. He is the Technical Program Co-Chair of the 2012 IEEE Symposium on Power Electronics and Machines in Wind Applications (PEMWA 2012) and was the Technical Program Co-Chair and Finance Co-Chair of the PEMWA 2009. He was the recipient of the 2010 National Science Foundation CAREER Award, the 2010 IEEE Industry Applications Society Andrew W. Smith Outstanding Young Member Award, the 2011 UNL Harold and Esther Edgerton Junior Faculty Award, and the 2011 UNL College of Engineering Edgerton Innovation Award. 